DOI: https://doi.org/10.46296/yc.v5i9edespdic.0137

\title{
LA INTELIGENCIA COLECTIVA Y SU INCIDENCIA EN LOS ECOSISTEMAS TECNOLÓGICOS DE APRENDIZAJE
}

\section{COLLECTIVE INTELLIGENCE AND ITS IMPACT ON TECHNOLOGICAL LEARNING ECOSYSTEMS}

\author{
Moreira-Choez Jenniffer Sobeida ${ }^{1}$; Zambrano-Alcívar María Viviana ${ }^{2}$ \\ ${ }^{1}$ Licenciada en Secretariado, Magíster en Administración de Empresas, Maestrando en \\ Educación, Mención Pedagogía de Entornos Digitales / Instituto de Posgrado de la Universidad \\ Técnica de Manabí, Doctoranda en Educación Superior, Universidad de Palermo (UP), \\ Argentina. Docente de la Facultad de Filosofía, Letras y Ciencias de la Educación de la \\ Universidad Técnica de Manabí. Portoviejo, Ecuador. Correo: jenniffer.moreira@utm.edu.ec. \\ ORCID ID: https://orcid.org/0000-0001-7172-7459 \\ ${ }^{2}$ Ingeniera en Sistema Computacional, Maestrando en Educación, Mención Pedagogía de \\ Entornos Digitales / Instituto de Posgrado de la Universidad Técnica de Manabí, Docente de la \\ Facultad de Filosofía, Letras y Ciencias de la Educación de la Universidad Técnica de Manabí. \\ Portoviejo, Ecuador. Correo: maria.zambrano@utm.edu.ec. ORCID ID: https://orcid.org/0000-
} 0001-5288-0247

\begin{abstract}
Resumen
El objetivo de la investigación se basó en analizar la incidencia de la inteligencia colectiva en los ecosistemas tecnológicos de aprendizaje, correlacionando los factores bióticos y abióticos bajo los que se desarrolla todo ecosistema, enfocándose específicamente en el ámbito de las TIC por medio de los sistemas Learning Management System - LMS, en reciprocidad con estudios sobre inteligencia colectiva llevados a cabo por Pierre Lévy y, darles una caracterización desde la visión de la informática, puntualizando en las herramientas que surgen con la inteligencia colectiva. Esta investigación se orientó en una metodología de investigación documental, de carácter cualitativo. El análisis de resultados y su discusión se sustentó en los estudios de Pierre Lévy y los aspectos sobre tendencias en innovación educativas, específicamente en la herramienta Moodle.
\end{abstract}

Palabras claves: aprendizaje, ecosistema, inteligencia colectiva, tecnología.

\begin{abstract}
The objective of the research was based on analyzing the incidence of collective intelligence in technological learning ecosystems, correlating the biotic and abiotic factors under which every ecosystem develops, focusing specifically on the field of ICT through Learning systems. Management System - LMS, in reciprocity with studies on collective intelligence carried out by Pierre Lévy and, give them a characterization from the computer science point of view, specifying the tools that arise with collective intelligence. Said research was oriented on a documentary research methodology, of a qualitative nature. The analysis of the results and their discussion was based on the studies of Pierre Lévy and the aspects of trends in educational innovation, specifically in the Moodle tool.
\end{abstract}

Keywords: learning, ecosystem, collective intelligence, technology.

Información del manuscrito:

Fecha de recepción: 29 de octubre de 2021.

Fecha de aceptación: 08 de diciembre de 2021.

Fecha de publicación: 17 de diciembre de 2021. 


\section{Introducción}

Las grandes distancias existentes entre la tecnología y las metodologías docentes, provoca que los nuevos avances tecnológicos no tengan fácil su integración en los contextos y practicas metodológicas implementados, lo que se traduce, en que los métodos educativos aplicados no respondan a las demandas de la sociedad ni al potencial transformador de la tecnología para la mejora del aprendizaje (García-Peñalvo et al., 2015). En contexto, según Váldez (2015) la sociedad del conocimiento es una sociedad del saber; en la cual se concibe dicho saber cómo la habilidad que se tiene para hacer una cosa; mientras que el conocimiento abarca una comprensión más global o analítica de esta cosa. El auge tecnológico que ha surgido en las últimas décadas ha fomentado que la integración en espacios educativos marque un ciclo donde prevalece la utilización de métodos implicados con actualización tecnológica, con el objeto de delinear enfoques pedagógicos que converjan con las necesidades de la sociedad del conocimiento.
En función a ello, se deriva la conceptualización sobre la inteligencia colectiva, la cual está fundamentada en la afirmación y la reciprocidad entre los individuos. Con esto, el fijarle una fecha de origen a la inteligencia colectiva, repercutiría en adentrarse en los orígenes de la humanidad, detallando sus aspectos evolutivos, los cuales engloban ámbitos como las revoluciones culturales representadas por la autoconservación de los símbolos, la aparición del alfabeto, la imprenta y la algorítmica.

Asimismo, señala Zuluaga-Duque (2015), que la "inteligencia colectiva no implica exclusivamente una característica cognitiva, sino que también implica una característica emotiva". Esta, proporciona un proceso bidireccional del conocimiento, haciendo que la sociedad se apropie del mismo. Con ello, el referido autor, señala que la inteligencia colectiva representa un cumulo de "conocimientos científicos, tecnológicos, técnicos, religiosos, metafísicos, humanistas, artísticos e ideológicos".

A tal efecto, la distribución de la inteligencia en todos los diferentes 
ámbitos de la sociedad, reconoce que el desconocimiento de un individuo de determinado tema, puede repercutir en implicaciones de modificar la absorción de conocimiento y de destrezas ante determinada situación. Tanto es así, que la inteligencia colectiva se deriva en espectros éticos y estéticos, que llegan a ser tan representativos como la tecnología y el comportamiento de una organización; lo que orienta a la alimentación de los vínculos sociales en función del conocimiento que se adquiere.

Para que esto se lleve a cabo, se debe fundamentar el espectro ambiental en el que se desarrolla la inteligencia colectiva, tal como lo son los ecosistemas, los cuales de acuerdo con Badii, Landeros \& Cerna (2007) son definidos como:

"un conjunto de sistemas complejos sustituidos por numerosos componentes; seres vivos y ambiente físico; que interactúan en diferentes escalas temporales y espaciales, permitiendo el intercambio entre la energía y la materia, y como consecuencia de estas interacciones, poseen una estructura y función específicas, por lo que representan algo más que la simple suma de cada uno de sus componentes"

Los ecosistemas están conformados a nivel medioambiental en factores abióticos y factores bióticos.

Existen un gran número de definiciones de ecosistema, pero todas hacen referencia a tres elementos principales como lo son el medio físico o factores abióticos; las relaciones entre los organismos y; los organismos o factores bióticos. Los elementos abióticos dependen del agua, la cual es el mecanismo fundamental para la vida $y$, para mantener el equilibrio ecológico de nuestro planeta. Mientras que los elementos bióticos, lo representa la biodiversidad que prolifera en la tierra (vida animal y vegetal).

Asimismo, García-Holgado \& García-Peñalvo (2013), señalan que, en el ámbito de la tecnología, "los componentes de software $y$ las personas que forman el ecosistema tecnológico, se corresponden con los factores bióticos; los flujos de información representan 0 establecen las relaciones entre los organismos y; los factores abióticos 
son los elementos que permiten el funcionamiento del ecosistema (hardware, conexión de red, etc.)". De ello, se puede referir que un ecosistema tecnológico se conforma por un estrato de usuarios y equipamientos tecnológicos que interactúan entre sí, por medio de flujos de información en un ambiente físico que facilita el respaldo de la información.

En concordancia, Gartner Research (2020) establece que los ecosistemas digitales se pueden definir como "un grupo interdependiente de empresas, personas y/o cosas que comparten plataformas digitales estandarizadas para un propósito mutuamente beneficioso, como ganancia comercial, innovación o interés común".

Estos se interrelacionan con los ecosistemas de aprendizaje, tal como lo señalan McPherson y Nunes (2008) quienes detallan que

"un ecosistema de aprendizaje es la suma de una comunidad de aprendices y formadores, de recursos para el aprendizaje (vídeo, audio, texto, ...), de principios y métodos (adaptativo, activo), de sistemas y procesos (internet, multimedia, web semántico), y administración de recursos para el aprendizaje (adquiere, organiza, recupera, reutiliza)".

Asimismo, García-Peñalvo et al. (2015) definen a los ecosistemas tecnológicos como "una comunidad donde métodos educativos, políticas, reglamentos, aplicaciones $y$ equipos de trabajo, pueden coexistir de forma que sus procesos están interrelacionados y su aplicación se basa en los factores físicos del entorno tecnológico". Por lo que dicha comunidad, representa toda una matriz virtual donde se establecen las bases para la generación de conocimiento y el desarrollo de la inteligencia en sus diferentes expresiones.

En tal sentido, se fundamenta como objetivo de la investigación el analizar la incidencia de la inteligencia colectiva en los ecosistemas tecnológicos de aprendizaje, para la cual se plantea la investigación desde un enfoque cualitativo, basado en una revisión documental; para la cual fue necesario recurrir a la recopilación de información de fuentes primarias 
documentales y de páginas web sobre los términos relacionados con la inteligencia colectiva y los ecosistemas tecnológicos de aprendizaje.

\section{Metodología}

El trabajo de investigación se formuló bajo un enfoque cualitativo, donde para la sustentación de la información en referencia a la inteligencia colectiva y su interacción en el ámbito de los ecosistemas tecnológicos de aprendizaje, se fundamentó en estudios llevados a cabo por Pierre Lévy, durante su trayectoria en el campo de la inteligencia colectiva y del trabajo de la Dra. Letizia Vaca sobre la gestión del ecosistema tecnológico en entornos digitales de aprendizaje. Para esto se utilizó la técnica de investigación documental, la cual facilitó la recolección de datos de origen secundario.

\section{Desarrollo}

\section{$\checkmark$ Ecosistema}

De acuerdo con Christopherson (1996), un ecosistema "es una unidad natural formada por todas las plantas, animales, y microorganismos que interactúan conjuntamente con todos los factores abióticos en su entorno". De esta definición, se asocia hacia áreas específicas de las ciencias, como lo es la biología, entendiendo que un ecosistema constituye una comunidad, en la cual seres vivos interactúan entre sí y con su entorno físico; por lo que Wilkinson en el año 2002, propuso el concepto de ecosistema de aprendizaje (Figura 1) estableciendo un paralelismo con un ecosistema biológico.

Tal es así, que Kirkham y Wood (2009) estableciendo en su estudio que "una aproximación típica a un ecosistema vincula organizaciones a herramientas compartidas y a tecnologías". Por lo que, de ello, se direcciona que el mejoramiento de los ecosistemas de apoyo al aprendizaje, se basa en los métodos con los que se cuenta, convergiendo en función de colaboraciones existentes y la evolución de los procesos.

De igual manera, Sánchez (2020) señala que la perspectiva ecosistémica para el aprendizaje es actualmente una tendencia que está promoviendo innovaciones educativas - tecnológicas basadas en nuevos paradigmas. 
Figura 1. Ecosistema de aprendizaje.

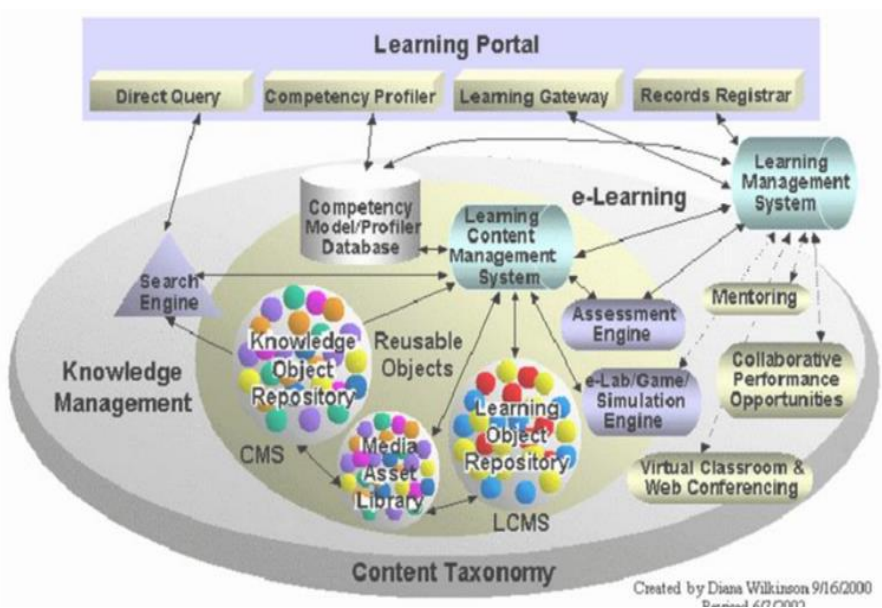

Fuente: En la figura se muestra la estructura del ciclo de un ecosistema de aprendizaje. Tomado de The Intersection of Learning Architecture and Instructional Design in e-Learning, por Wilkinson, 2002.

Es por ello, que de acuerdo con Fidalgo-Blanco et al (2015) señalan que la gestión del conocimiento y, más concretamente de los procesos de enseñanza - aprendizaje dentro de las instituciones y empresas, ha provocado la evolución de los sistemas de información tradicionales en lo que actualmente se denominan ecosistemas tecnológicos. Así, se tiene que una diferencia de los ecosistemas tecnológicos frente a los sistemas de información, es la capacidad de mezclarse con los de dispositivos tecnológicos para facilitar accesibilidad a los usuarios (Figura 2).

Figura 2. Ecosistema tecnológico de aprendizaje

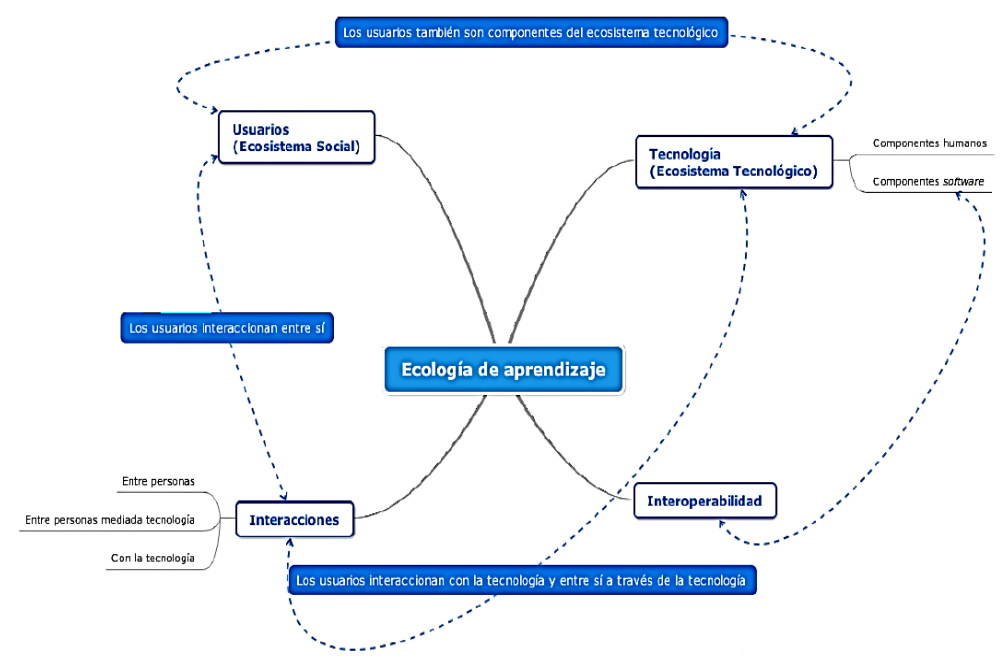

Fuente: En la figura se muestra los componentes e interacciones en un ecosistema tecnológico de aprendizaje. Tomado de Ecosistemas tecnológicos universitarios, por García-Peñalvo, 2016. 


\section{Características de un ecosistema} de aprendizaje

Tiwana (2014), señala cuatro propiedades básicas deseables que se encuentran correlacionadas en los ecosistemas de aprendizaje, como lo son:

- Simplicidad.

- Resiliencia.

- Sostenibilidad.

- Capacidad de evolucionar.

Ello corresponde a la arquitectura de un ecosistema, la cual según GarcíaHolgado \& García-Peñalvo (2013), relacionan que está confeccionada en cinco ítems que direccionan a los ecosistemas tecnológicos, como lo son: la gestión de los usuarios, la gestión de los datos y de la información, los componentes de social media, la capacidad de integración entre los diferentes componentes del ecosistema y, la capacidad de evolución de los componentes y/o dispositivos.

Asimismo, García-Peñalvo (2016) señala una serie de atributos que se deben manejar para el diseño de la arquitectura de un ecosistema de aprendizaje, en donde se puntualiza

en: la integración, la interoperabilidad, la evolución de los componentes y, la definición de la arquitectura que funge de respaldo. Esto sirve de sustento para la aplicación de la eficiencia de la gestión de los recursos de aprendizaje, en donde se deben manejar unos puntos que garantizan el éxito de los atributos, como los son: la disponibilidad y accesibilidad, la calidad, la relevancia y, la reusabilidad.

\section{Ecosistemas tecnológicos de aprendizaje}

Según Chang \& West (2006), las acepciones sobre los ecosistemas tecnológicos varían, pero se converge en que "hay una clara relación entre las características de un ecosistema natural y un ecosistema tecnológico en cualquiera de sus variantes". Con él pasar de los años, las tecnologías del aprendizaje, se ha puntualizado que las tecnologías utilizadas en el ámbito educativo, no han sido aprovechadas de manera eficiente, por lo que de sus potencialidades se puede relacionar que se pueden anexar al proceso de aprendizaje de los individuos, para hacerlas 
participes de la sociedad del conocimiento.

Asimismo, la expansión que representan las tecnologías en la cotidianidad, genera un proceso de enseñanza - aprendizaje por medio de la transferencia de conocimiento $y$, de su apropiación por parte del colectivo.

Con ello, se tiene que, para los estudiantes un ecosistema tecnológico de aprendizaje (Figura 2) debe considerar las siguientes características:

- Facilitar el acceso a la plataforma: debe ser sencillo y que motive la participación.

- $\quad$ Accesibilidad a los contenidos y actividades con pocos clics.

- Posibilidad de interacción con compañeros.

- Facilidad de acceso al docente para consultas y dudas.

Desde el punto de vista de la administración y gestión debe tenerse en cuenta la posibilidad de expansión, tanto de crecimiento en cantidad de estudiantes como de cursos y recursos educativos y, la facilidad para solucionar problemas tecnológicos y administrativos.

En base a ello, Avgeriou et al (2003) los Learning Management System (LMS) facilitan instrumentos que expanden y proporcionan respaldo a las acepciones tradicionales de clase, ya que se basan en colaborar con los docentes, por medio del apoyo en las actividades administrativas y de gestión relacionadas con el proceso de aprendizaje.

Por tanto, los LMS (Tabla 1) están dirigidas a la interacción entre docentes y estudiantes, pero con relativa rigidez de los flujos de información, limitando la interactividad. Tanto es así, que las carencias de los LMS, los profesores y estudiantes tratan de complementarlos con otras herramientas y servicios, ya sean proporcionados por la institución o libremente accesibles en la red, gracias a la oferta tecnológica al alcance de cualquier persona. 
Tabla 1. Herramientas Learning Management System - LMS.

\begin{tabular}{|c|c|c|c|c|c|}
\hline LMS & $\begin{array}{l}\text { Programado } \\
\text { en }\end{array}$ & Base de Datos & LMS & $\begin{array}{l}\text { Programado } \\
\text { en }\end{array}$ & Base de Datos \\
\hline Atutor & $\mathrm{PHP}$ & $\begin{array}{l}\text { MySQL, } \\
\text { MariaDB } \\
\text { PostgreSQL }\end{array}$ & Moodle & $\mathrm{PHP}$ & MySQL \\
\hline Cacus & CML & MySQL & & & \\
\hline Canvas & Ruby & $\begin{array}{l}\text { PostgreSQL } \quad 0 \\
\text { SQLite }\end{array}$ & OpenOlat & Java & $\begin{array}{l}\text { MySQL, } \\
\text { MariaDB } \quad \text { o }\end{array}$ \\
\hline Chamilo & PHP & MySQL & & & PostgreSQL \\
\hline Claroline & PHP & MySQL & Opingo & PHP & MySQL \\
\hline Forma & PHP & MySQL & & & \\
\hline ILIAS & PHP & MySQL & & & \\
\hline Mahari & PHP & $\begin{array}{l}\text { MySQL, } \\
\text { MariaDB } \\
\text { PostgreSQL }\end{array}$ & Sakai & Java & $\begin{array}{l}\text { MySQL } \\
\text { Oracle }\end{array}$ \\
\hline
\end{tabular}

Nota: Esta tabla muestra la clasificación de las herramientas LMS, en función a su programación. Tomado de Gestión del ecosistema tecnológico en entornos digitales de aprendizaje, por Vaca, 2020. Según García Peñalvo et al (2015), el diseño de un ecosistema tecnológico de aprendizaje y gestión educativa "está orientado a dotar de las herramientas tecnológicas que permitan apoyar a todos los procesos vinculados a la actividad propia de un contexto educativo".

En el mismo orden de ideas, un 3) que afectan el sistema educativo ecosistema de aprendizaje debe estar dirigida a responder a unas características estratégicas (Figura dentro del ámbito de sus procesos (estratégicos, fundamentales y de apoyo).

Figura 3. Características estratégicas de un ecosistema tecnológico de aprendizaje

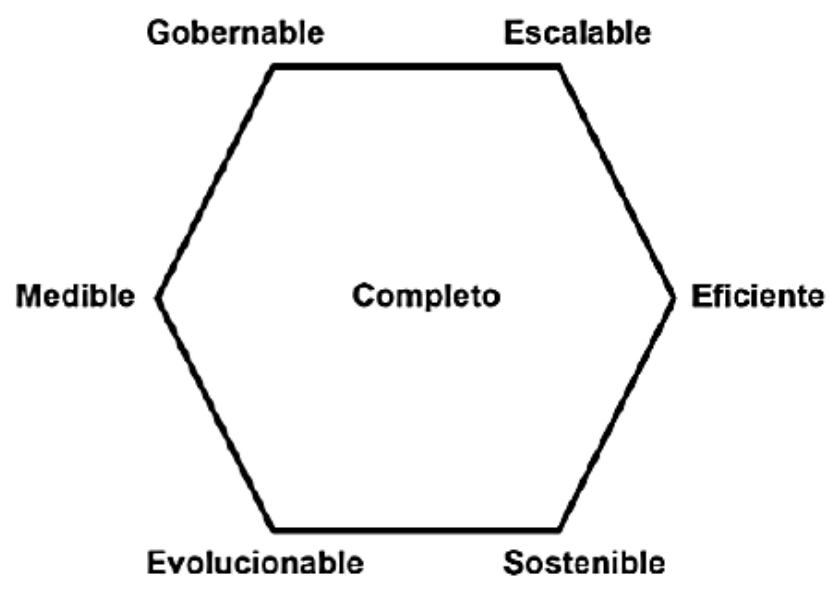

Nota: En la figura se muestran las estrategias a seguir para establecer un ecosistema de aprendizaje. Tomado de Ecosistemas tecnológicos de aprendizaje y gestión educativa. Características estratégicas para un diseño eficiente, por Martí, R., Gisbert, M. y Larraz, V., 2018. 
Un factor crítico para el diseño eficiente de un ecosistema tecnológico de aprendizaje, es la participación de todos los agentes implicados (profesorado, equipos directivos, responsables a nivel de gobierno, alumnos, familias $y$, también, responsables y empresas proveedoras de tecnología).

\section{Herramientas en la construcción de inteligencia colectiva}

De acuerdo con Váldez (2015), las contextualizaciones principales para las herramientas que hacen parte en el proceso de construcción de inteligencia, son las siguientes:

- Aprendizaje colaborativo: la formación también puede beneficiarse de la inteligencia colectiva. El antiguo modelo vertical profesor/alumno puede ser sustituido por comunidades de aprendizaje donde los propios estudiantes aportan materiales. Moodle es una plataforma abierta de aprendizaje que permite crear estas comunidades.

- Etiquetaje colectivo: es un sistema en el que los usuarios etiquetan información de manera que sea más fácil su clasificación, archivo y consulta.

- Votación y rankings colectivos: se trata de un sistema inspirado en webs que permite que los usuarios destaquen información mediante votaciones. De esta manera la información que se prioriza es la que interesa a la mayoría de los usuarios y no la que se cree desde alguna instancia que interesa. En las organizaciones la votación colectiva permite conocer la opinión de los trabajadores o los clientes, priorizar actuaciones y filtrar información significativa.

- Wikis: es un documento elaborado de manera colectiva y simultánea por parte de varias personas. La filosofía detrás del concepto de wiki surge de la revisión entre iguales (peer review) de las revistas científicas: si suficientes personas leen un texto, aumenta la posibilidad de detectar y corregir los errores. En las organizaciones, el uso de wikis en la intranet puede permitir consolidar los conocimientos existentes, evitar la reduplicación de esfuerzos y compartir buenas prácticas. 
Para la implementación de los procesos de generación de inteligencia colectiva en los ambientes de aprendizaje, se deben complementar premisas de las áreas de acción. Por ello, la tendencia del proceso de aprendizaje se enfoca a los entornos colaborativos y de apoyo, buscando la construcción de saberes de manera colectiva. Por otro lado, la necesidad de internalizar la información y su fusión con los conocimientos previamente adquiridos se enfocan hacia la apropiación social del conocimiento.

Con ello, se enfoca que las tecnologías de información y comunicación en los ambientes educativos, son pertinentes para el reconocimiento de la sociedad del conocimiento en base al apoyo tecnológico.

En tal sentido, Lévy (2004) señala que:

"algunos dispositivos que permiten desarrollar la inteligencia colectiva son los instrumentos de vínculo social encaminados a incluir al otro, a compartir $y$ aprehender con él; el aprovechamiento de los medios masivos de comunicación que buscan efectivamente poner en común las construcciones simbólicas de todos los hombres; los sistemas que persiguen como fin desarrollar en el hombre su autonomía y, el incremento de las ingenierías semióticas que posibiliten que los hombres puedan enriquecer sus competencias, acceder a los datos y apropiarse de la potencia simbólica de la humanidad".

De igual manera, Lévy (2015) define a la inteligencia colectiva "como el resultado de una colaboración abierta distribuida en el medio algorítmico donde las tecnologías se hacen invisibles y dan paso a la generación, conexión y distribución del conocimiento creativo en una comunidad de aprendizaje".

Para el referido autor, la inteligencia personal implica una inmersión en el "big data", aplicando estrategias de conectividad y selección para la búsqueda de información, planteando el análisis de datos y la categorización y protección de la información. De ello, se señala que el caos en el procesamiento de la información, compromete el cómo, dónde y cuándo aprender, lo que socava el surgimiento de estrategias 
para el procesamiento de la información.

\section{Resultados}

En función a los estudios llevados a cabo por Lévy (2007), se fundamenta que la inteligencia colectiva facilita enfoques integradores, generando una simbiosis entre el desarrollo de la sociedad del conocimiento y el del desarrollo humano. Asimismo, el plasmar las especificaciones de la denominada economía de la información y el metalenguaje, como requisitos para las finalidades de la sociedad del conocimiento, por lo que la convergencia entre el capital ético, práctico, biofísico, social, de comunicación y epistémico, dan lugar a flujos de información, que indican la interdependencia de los diversos aspectos de desarrollo humano, lo que define la "inteligencia colectiva" de una comunidad. El ciberespacio se presenta entonces como un instrumento de mediación entre el saber colectivo y la sociedad humana en desarrollo.

La construcción del ecosistema de conocimiento inicia con el diálogo, por lo que hay programas de software que se desarrollan para posibilitar diálogos construidos argumentativamente, pero no están integrados a otras herramientas colaborativas que presentan servicios web integrados y, que ya se han liberado para su uso extensivo. Aunque el hecho de no contar con un software que se enfoque en el desarrollo de competencias comunicativas y estrategias dialógicas de manera precisa, actualmente los entornos y aplicaciones disminuyen las barreras para el desarrollo de la inteligencia colectiva.

Cada nivel del ecosistema de conocimiento puede ser soportado por entornos y herramientas que apuntan a la construcción del ecosistema en su conjunto (Figura 4). Por lo que el desarrollo de mapas mentales favorece la estructuración del producto colectivo a desarrollar. 
Figura 4. Ecosistema del conocimiento y tipos de soporte tecnológico

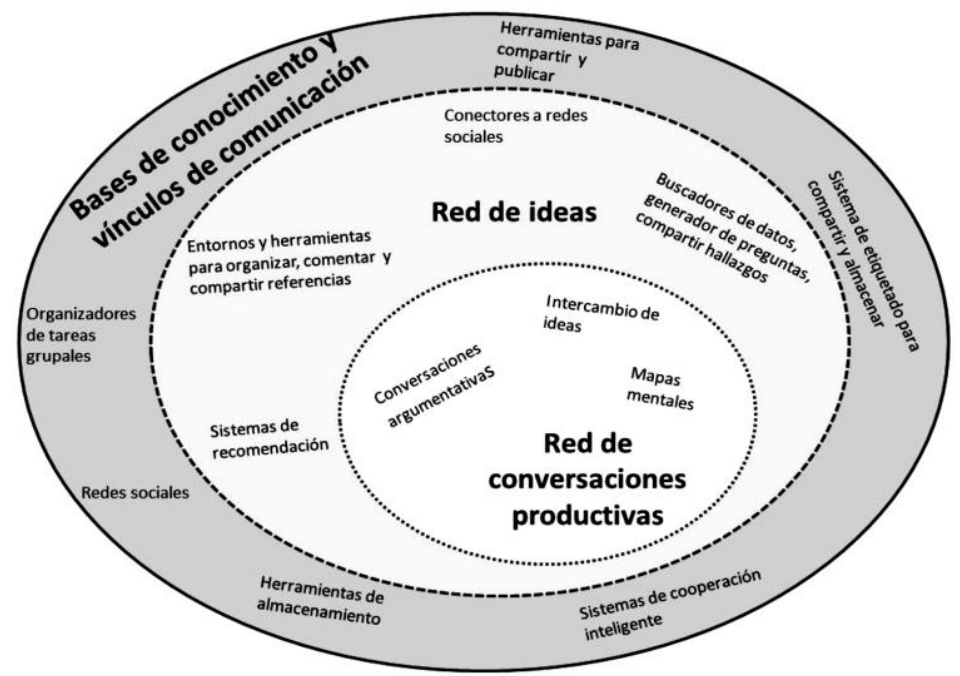

Nota: En la figura se muestran la composición del ecosistema del conocimiento y el tipo de soporte tecnológico que se debe cumplir para que se genere. Tomado de Ecosistemas tecnológicos de aprendizaje y gestión educativa. Características estratégicas para un diseño eficiente, por Chan, M., 2015.

La inteligencia colectiva, esta arraiga como uno de los ejes fundamentales de las tendencias actuales en el ámbito de la innovación educativa (Figura 5) en diferentes grados de implantación. En donde Moodle se ha convertido en una tendencia en innovación educativa. En relación a ello, Fidalgo-Blanco et al (2019) señalan que Moodle es "una tecnología dentro de la categoría LMS, cuya principal innovación es que se basa en software libre y esta característica, unida a su funcionalidad, hace que se introduzca rápidamente en el sector formativo".

Figura 5. Tendencias actuales en innovación educativa

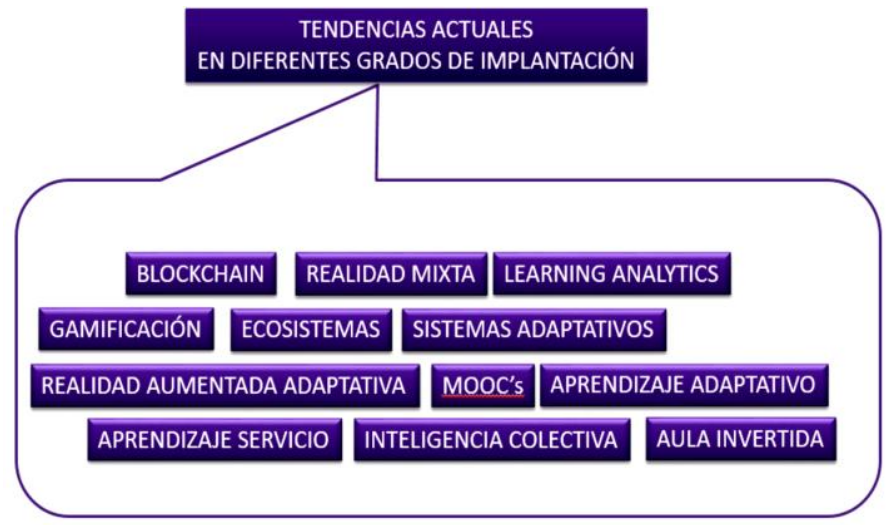

Nota: En la figura se plasman las tendencias en innovación educativa que están proliferando en la actualidad. Tomado de Tendencias de innovación educativa con Moodle: Llevando el cambio metodológico al aula, por Fidalgo-Blanco, A., Sein Echaluce, M., García-Peñalvo, F., 2019. 
Actualmente es uno de los sistemas LMS/LCMS más utilizados en las universidades. A pesar de este gran impacto Moodle, como tal, fue una innovación en los primeros años, pero actualmente no se puede considerar como innovación. Sin embargo, una cosa es que Moodle no se considere desde el punto de vista de la tecnología una innovación y otra que cosa es que realmente no lo pueda ser. Para comprobar si
Moodle (Figura 6), o cualquier otra tecnología consolidada, se puede utilizar en innovación educativa, y por tanto ser una tecnología se debe relacionar la tecnología con tres características de la innovación educativa: Tipos de innovación educativa en función del contexto de participación del profesorado, los tipos de innovación y la comestibilidad de la innovación educativa.

Figura 6. Moodle en la innovación educativa con LMS

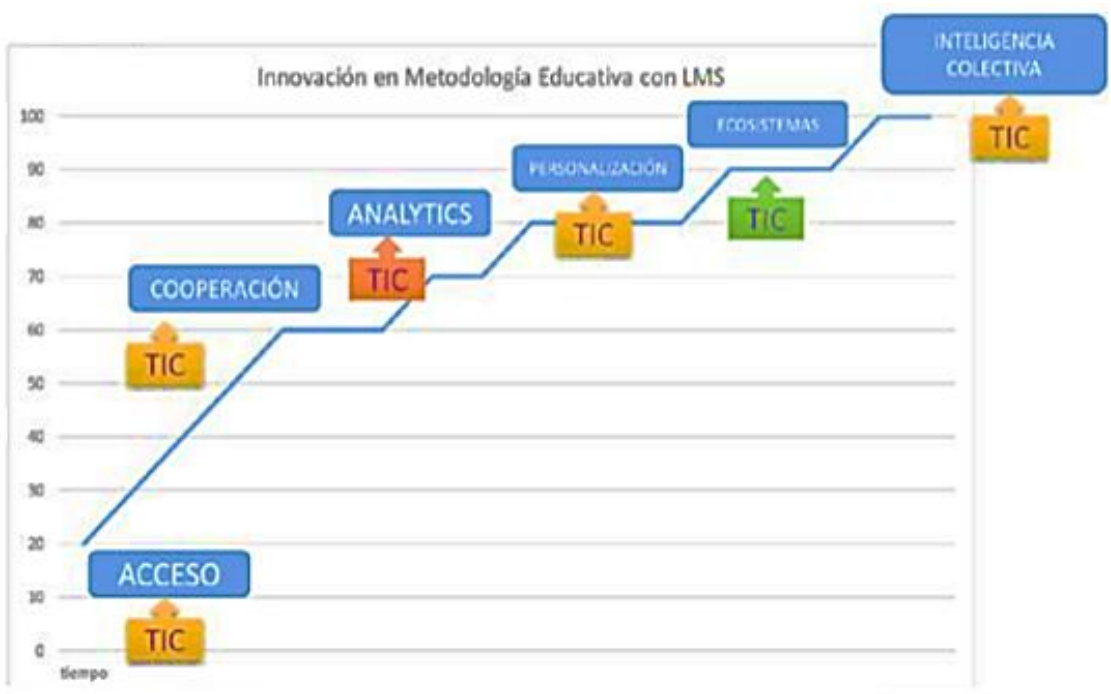

Nota: En la figura se presentan la escalación en tendencias en metodología educativa aplicadas con herramientas LMS. Tomado de Tendencias de innovación educativa con Moodle: Llevando el cambio metodológico al aula, por Fidalgo-Blanco, A., Sein Echaluce, M., García-Peñalvo, F., 2019.

\section{Discusión}

De acuerdo con la información documental recopilada para el desarrollo de la investigación, la selección y análisis de datos direcciona la determinación del aprendizaje, enfocando la atención en un determinado tema para la toma de decisiones. Por ello, la inteligencia personal, surge con una inmersión en el "big data", aplicando estrategias conectivas y a su vez selectivas en la búsqueda de 
información, planteando hipótesis y, analizando los datos.

La inteligencia colectiva se deriva de lo anterior, más las acciones de un aprendizaje colaborativo, en donde se visualiza que cuan mayor capital cognitivo se encuentre en el ciberespacio, se incrementa el acceso a la información para la apropiación del conocimiento.

Por ende, la relevancia de la generación de redes y conexiones en la sociedad de la información, incide directamente en la sociedad del conocimiento, lo que repercute en una transformación total de la dinámica de los entornos de los ecosistemas tecnológicos de aprendizaje, en donde a nivel de la innovación educativa Moodle se ha puesto en la primera línea como una herramienta eficaz para la interactividad del conocimiento colectivo.

\section{Conclusiones}

La denominada cultura digital, es protagonista de los cambios culturales, cambiando los paradigmas en todos los ámbitos de la sociedad. Ahora bien, con la digitalización, el conocimiento se ha propagado, ya que las redes están inundadas de información.

El hecho de que lo conectivo y lo colectivo se presenten con la característica de que el acceso a la información que corre por la red es proveniente de diferentes entornos y es ubicua, se puede acceder a ella casi en cualquier parte y momento. Dicha conectividad y colectividad tienen implicaciones pedagógicas en el área del aprendizaje, la enseñanza, la construcción de la ciencia, la función de la escuela y la construcción de procesos formativos propios de los ciudadanos de la cibercultura (entre otros).

Las personas manejan cada vez con más facilidad las tecnologías de información y comunicación, satisfaciendo necesidades y surgiendo nuevas a su vez. Por ello, se debe tener en cuenta con la inteligencia colectiva en un entorno de ecosistemas tecnológicos de aprendizaje, debe responde a unas características en cuanto a su arquitectura en la gestión educativa, como lo son: la simplicidad, la resiliencia, la sostenibilidad y la capacidad de evolucionar. 


\section{Bibliografía}

Avgeriou, P., Papasalouros, A., Retalis, S. y Skordalakis, E. (2003). Towards a Pattern Language for Learning Management Systems.

Badii, M., Landeros, J. y Cerna, E. (2007). Papel de los ecosistemas en la sustentabilidad. Monterrey, México.

Baltzersen, R. (2019). CulturalHistorical Perspectives On Collective Intelligence. Patterns In Problem Solving and Innovation.

Chan, M. (2015). Comunidades y redes académicas en los ecosistemas de conocimiento. La Plata, Argentina. Universidad Nacional de La Plata.

Chang, E. y West, M. (2006). Digital Ecosystems A Next Generation of the Collaborative Environment. 8th International Conference on Information Integration and Web-based Application \& Services.

Christopherson, R. (1996). Geosystems: An Introduction to Physical Geography. Prentice-Hall, Englewood Cliffs.

Cobo, C. y Moravec, J. (2011). Aprendizaje invisible. Hacia una nueva ecología de la educación. Edicions de la Universidad de Barcelona.

Drago, W, Peltier, J. y Sorensen, D. (2002). Course content or instructor: which is more important in online teaching? Management

Research News.

Drennan, J., Kennedy, J. Pisarki, A. (2005). Factors affecting student attitudes toward flexible online learning in management education. The Journal of Educational Research.

Fidalgo-Blanco, A., Sein Echaluce, M., García-Peñalvo, F. (2019). Tendencias de innovación educativa con Moodle: Llevando el cambio metodológico al aula. Bogotá, Colombia. Conferencia MoodleMoot.

García-Holgado, A. y GarcíaPeñalvo, F. (2013). Definición de ecosistemas de aprendizaje independientes de plataforma. Salamanca, España. Universidad de Salamanca.

García-Peñalvo, F., HernándezGarcía, A., Conde-González, M., Fidalgo-Blanco, A., SeinEchaluce Lacleta, M., AlierForment, M., Llorens-Largo, F. y Iglesias-Pradas, S. (2015). Mirando hacia el futuro: Ecosistemas tecnológicos de aprendizaje basados en servicios. Madrid, 
España. Actas del III Congreso Internacional sobre Aprendizaje, Innovación y Competitividad - CINIAC.

García-Peñalvo, F. (2016). En clave de innovación educativa. Construyendo el nuevo ecosistema de aprendizaje. Salamanca, España. Universidad de Salamanca.

Gartner Research (2020). Ecosistemas digitales. Medellín, Colombia. Academia Pragma.

Kirkham, T., Wood, S., Winfield, S., Coolin, K. y Smallwood, A. (2009). An ecosystem for user centric learning: revolution or evolution? InProceedings of the International Conference on Management of Emergent Digital EcoSystems.

Lévy, P. (2004). Inteligencia colectiva. Washington, D.C. Organización Panamericana de la Salud.

Lévy, P. (2007). Cibercultura. Informe al Consejo de Europa. Barcelona. Anthropos Editorial.

Lévy, P. (2015). Inteligencia colectiva para educadores. Organización de Estados Iberoamericanos - OEI. Conferencia de Pierre Lévy.

Martí, R., Gisbert, M. y Larraz, V. (2018). Ecosistemas tecnológicos de aprendizaje y gestión educativa. Características estratégicas para un diseño eficiente. EDUTEC, Revista Electrónica de Tecnología Educativa.

McPherson, M. y Nunes, J. (2008). Critical issues for e-learning delivery: what may seem obvious is not always put into practice. Journal of Computer Assisted Learning.

Peltier, J., Schibrowsky, J. y Drago, W. (2007). The interdependence of the factors influencing the perceived quality of the online learning experience: a causal model. Journal of Marketing Education.

Tiwana, A. (2014). Platform ecosystems: aligning architecture, governance and strategy. Morgan Kaufmann Publishers. Elsevier.

Uden, L. (2007). The Three E's: elearning, e-knowledge and eecosystem for SMEs. Staffordshire University.

Vaca, L. (2020). Gestión del ecosistema tecnológico en entornos digitales de aprendizaje. Manabí, Ecuador. Universidad Técnica de Manabí.

Váldez, Y. (2015). Contextualización de la inteligencia colectiva en los espacios de aprendizaje. Aragua, Venezuela. Universidad Bicentenaria de 
Aragua. IV Jornadas de Investigación.

Wareham, J., Fox, P. \& Cano-Giner, J. (2013). Technology Ecosystem Governance. Institute for Operations Research and the Management Sciences INFORMS.

Wilkinson, D. (2002). The Intersection of Learning Architecture and Instructional Design in e-Learning. $\mathrm{ECl}$ Conference on eTechnologies in Engineering Education: Learning Outcomes Providing Future Possibilities.

Zuluaga-Duque, J. (2015). La gestión crítica del conocimiento y la inteligencia colectiva y su relación con el desarrollo social. Medellín, Colombia. Universidad de Caldas. 